\title{
Japanese experience with hepatic resection of KRAS-mutated colorectal liver metastases
}

\author{
Junichi Shindoh ${ }^{1,2}$ \\ ${ }^{1}$ Hepatobiliary-Pancreatic Surgery Division, Department of Gastroenterological Surgery, Toranomon Hospital, Minato-ku, Tokyo, Japan; ${ }^{2}$ Okinaka \\ Memorial Research Center for Adult Diseases, Tokyo, Japan \\ Correspondence to: Junichi Shindoh, MD, PhD. Hepatobiliary-Pancreatic Surgery Division, Department of Gastroenterological Surgery, Toranomon \\ Hospital, 2-2-2 Toranomon, Minato-ku, Tokyo 105-8470, Japan. Email: shindou-tky@umin.ac.jp.
}

\begin{abstract}
R A S$ mutation is a well-known prognostic marker predicting patterns of recurrence and survival after resection of colorectal liver metastases (CLMs). However, there has been scarce evidence regarding the optimal choice of treatment for RAS-mutated CLMs. Indeed, RAS-mutated CLMs are at high risk of lung metastases which may preclude curative-intent treatment at the time of recurrence. Nevertheless, aggressive surgical approach using repeated resection for recurrent lesions may have prognostic advantage regardless of the RAS mutational status. As such, basic management of CLMs including careful work-up, preoperative chemotherapy for oncologically unfavorable cases, curative-intent surgery, adjuvant chemotherapy for synchronous disease, and careful follow-up including thoracic scan for monitoring the lung metastases would be important to maximize the survival outcomes of patients with CLMs irrespective of $R A S$ mutational status.
\end{abstract}

Keywords: Colorectal liver metastases (CLMs); resection; chemotherapy; KRAS

Submitted Jun 17, 2019. Accepted for publication Aug 01, 2019.

doi: $10.21037 /$ cco.2019.08.07

View this article at: http://dx.doi.org/10.21037/cco.2019.08.07

\section{Introduction}

Multidisciplinary treatment approach including liver resection and chemotherapy has been reported as an effective strategy to improve the long-term outcomes of patients with colorectal liver metastases (CLM) (1). Because clinical courses of individual patients are highly influenced by their oncological and physical statuses (2-4), risk stratification before selecting a treatment is important to maximize the treatment outcomes especially for the patients with advanced disease.

In the era of precision medicine, emerging biologic markers such as RAS, BRAF, PIK3CA, TP53, or SMAD4 have been reported to be associated with oncological aggressiveness of tumors and treatment outcomes of patients with CLM (5-19). RAS mutation is a well-known prognostic marker predicting patterns of recurrence and survival $(12,14,20-22)$ after resection of CLM. Given that mutation in $R A S$ is reported to be associated with a risk of histologically narrower surgical margin $(23,24)$ and a higher risk of lung metastases $(12,14,22)$, intensive multidisciplinary treatment approach is usually required for the patients with $R A S$-mutated CLM.

In this review, optimal treatment approach for CLM is revisited and clinical features of $R A S$-mutated CLM is discussed based on our experience at a Japanese highvolume center.

\section{Significance of surgical resection for CLMs}

While CLM is a stage IV cancer by definition, optimal therapeutic intervention may prolong survival outcomes, and we can even expect "cure" in selected population through a multidisciplinary treatment approach. Several observational studies have reported that surgical resection is an effective treatment for improving long-term survival of patients with CLM $(1,25)$, and the current clinical guidelines have included "conversion to surgery" as a part 
Table 1 The preoperative score predicting disease-free survival after hepatic resection for colorectal liver metastases [adopted from Beppu et al. (28) with permission]

\begin{tabular}{|c|c|}
\hline Risk factors & Preoperative score \\
\hline \multicolumn{2}{|c|}{ Timing of liver metastases } \\
\hline Metachronous & 0 \\
\hline Synchronous & 3 \\
\hline \multicolumn{2}{|c|}{ Primary tumor LN status } \\
\hline Negative & 0 \\
\hline Positive & 3 \\
\hline \multicolumn{2}{|c|}{ Number of tumors } \\
\hline 1 & 0 \\
\hline $2-4$ & 4 \\
\hline$\geq 5$ & 9 \\
\hline \multicolumn{2}{|c|}{ Largest tumor diameter } \\
\hline$\leq 5 \mathrm{~cm}$ & 0 \\
\hline$>5 \mathrm{~cm}$ & 2 \\
\hline \multicolumn{2}{|c|}{$\begin{array}{l}\text { Extrahepatic metastatic disease } \\
\text { (at hepatectomy) }\end{array}$} \\
\hline No & 0 \\
\hline Yes & 4 \\
\hline \multicolumn{2}{|c|}{ CA19-9 level (before hepatectomy) } \\
\hline$\leq 100 \mathrm{U} / \mathrm{mL}$ & 0 \\
\hline$>100 \mathrm{U} / \mathrm{mL}$ & 4 \\
\hline
\end{tabular}

LN, lymph node.

of treatment algorithms for stage IV colorectal cancer (26). From a hepatobiliary surgeon's standpoint, however, the current clinical guidelines do not appropriately present surgical indication for CLM because cytoreduction (i.e., shrinkage of tumor) is not always a requisite condition for curative surgery.

\section{The requisite condition for cure of CLMs: indication criteria for surgery}

Theoretically thinking, if we can achieve complete removal of cancerous tissue, the patients will enjoy longterm survival. However, considering that surgery is a local therapy, the patients who will be benefitted from surgery should have localized disease. To meet the theoretical requisite for cure, surgical indication of CLM should be determined considering both "oncological" and "technical" standpoints (27).

Oncological resectability is dependent on the disease control probability with surgery. In many centers, clinical factors such as tumor size, number of nodules, timing of metastases (i.e., synchronous $v s$. metachronous), or presence of extrahepatic disease have been used as prognostic factors to predict the efficacy of surgery. A risk score established based on a Japanese nation-wide survey (28) has clarified prognostic weight of each clinical variable (Table 1). Considering that tumor number and synchronous presentation are two potent prognostic factors predicting worse survival outcomes, we usually perform a short course (4-6 cycles in general) preoperative chemotherapy for patients who have such unfavorable factors to confirm the oncological aggressiveness of the disease and curative potential with surgery.

Technical resectability criteria include (I) expectation of margin-negative resection and (II) sufficient volume of future liver remnant. For risk estimation of postoperative hepatic insufficiency, we have used original criteria, expanding conventional Makuuchi's criteria (29). In short, (I) serum bilirubin level $<2.0 \mathrm{mg} / \mathrm{dL}$, II) no or controllable ascites, and (III) estimated indocyanine green disappearing rate of the future liver remnant (ICG-Krem) $\geq 0.05$ which is calculated with ICG clearance test and three-dimensional volumetry of the liver (30). When a patient does not meet the volume criteria, portal vein embolization or two stage hepatectomy is considered.

\section{Preoperative chemotherapy and goal of the treatment}

Before starting discussion about the significance of preoperative chemotherapy, we should revisit the goal of chemotherapy for the patients with resectable or potentially resectable CLMs. As I mentioned above, tumor shrinkage is not always a goal of chemotherapy. Figure 1 illustrates the concept and purpose of preoperative chemotherapy. There is a wide range of patient group with marginally resectable disease between the definitely resectable and unresectable diseases. Given that marginally resectable diseases are technically resectable in most of the cases, while they have oncologically unfavorable factors for surgery, the purposes of chemotherapy should be different from "conversion" in such situation.

Conversion is the term used when unresectable disease is "converted" to potentially resectable disease usually through 


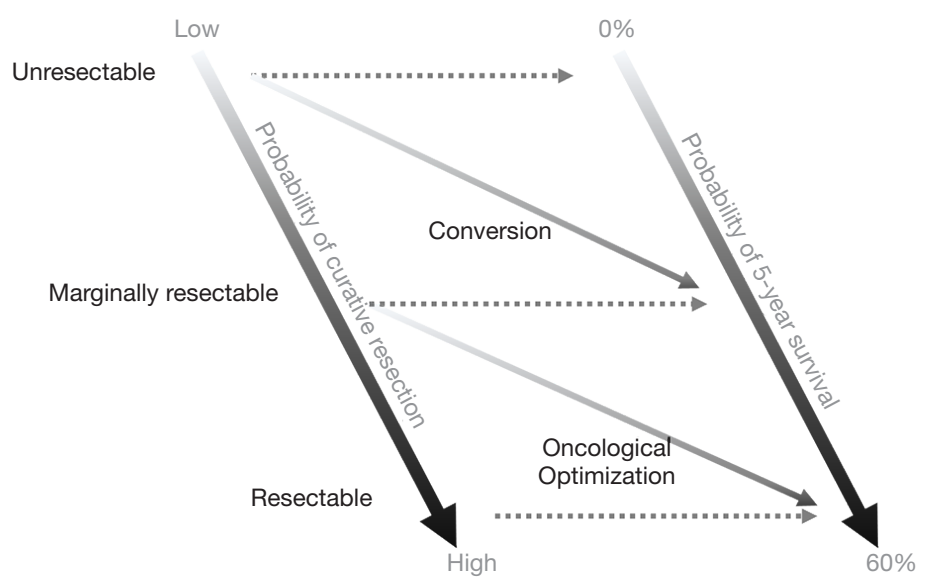

Figure 1 Concept and purpose of preoperative chemotherapy for colorectal liver metastases.

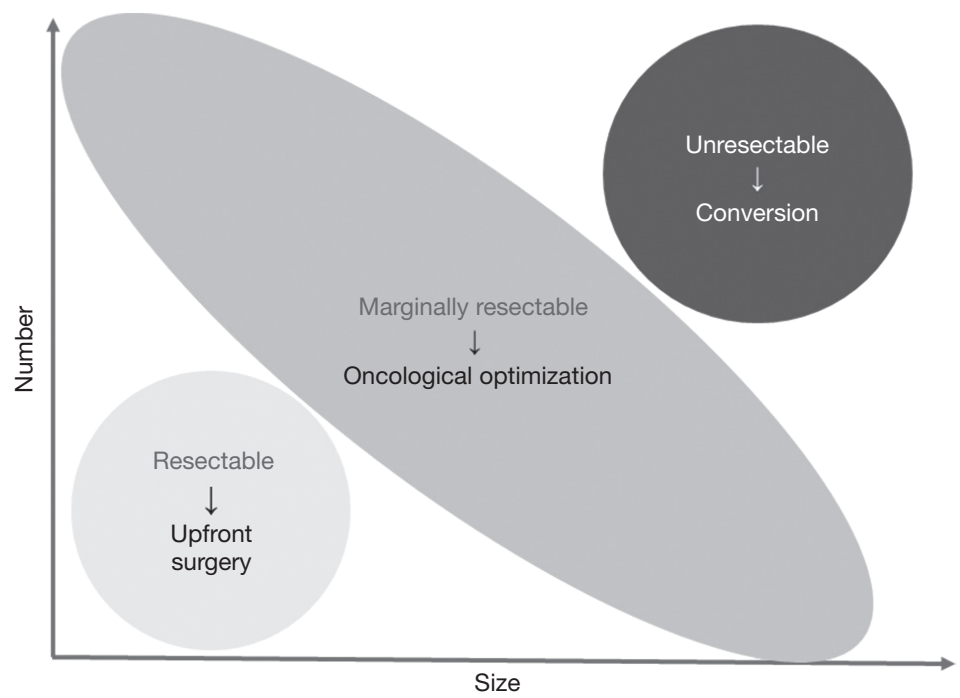

Figure 2 Resectability of colorectal liver metastases according to size and number of tumors.

a good response to chemotherapy. On the other hand, when a patient has CLMs which are technically resectable but oncologically unfavorable for surgery, we should wait and watch under a short course of chemotherapy to confirm the potential benefit of surgery. This group of patients actually have technically resectable disease and the term of "conversion" is not appropriate. Given that we select patients in whom surgical resections are oncologically meaningful in this process, we call this type of chemotherapy "oncological optimization (for surgery)".

Although maximum tumor shrinkage would be the primary goal for conversion to surgery among the initially unresectable diseases, the goal of treatment is relatively complex when treating patients with marginally resectable disease. Figure 2 illustrate the concept of "resectable", "marginally resectable", and "unresectable" diseases according to the size and numbers of tumors. Because marginally resectable tumors include quite heterogeneous population from solitary, huge tumor to multiple, tiny nodules, the main goal of the preoperative therapy should be different according to the oncological characteristics of individual tumors. Empirically, among patients with marginally resectable CLMs, shrinkage of tumor rarely changes planned surgical procedures, though the surgical maneuver may become less technically demanding. Medical oncologists tend to think that surgical procedure would be 
less invasive based on the dogma that degree of resection can be smaller as the tumors shrink with chemotherapy. Several studies have shown that volume of the normal liver parenchyma does not increase and sometimes shrinks with evidence of decreased hepatic functional reserve (31-33). Considering that prolonged chemotherapy does not improve pathologic response rate (34) and may induce chemotherapy-associated liver injury (35-37) length of preoperative chemotherapy should be short as possible when durable response is observed. Furthermore, it has been reported that regardless of the presence of sizebased response, long-term outcomes are better when pathological (38) and/or radiological morphologic response (39-41) is observed. Suboptimal pathological response and morphologic response are reportedly associated with presence and wide distribution of microsatellite lesions surrounding macroscopic CLMs (42), and these observations support that narrow margin status may not affect long-term outcomes when pathological or morphologic response is observed after chemotherapy (43).

\section{Basic surgical maneuver for CLMs}

Different from hepatocellular carcinoma that requires anatomic resection of the tumor-bearing portal territories (44), CLM can be cured basically with parenchymal sparing surgery. To expect long-term survival of patients with CLM, repeated resection of resectable recurrence is important and the clinical impact of repeated treatment for recurrence after resection has been reported as a concept of time-to-surgical failure (45). In this context, salvageability for recurrent lesions is important, and it has been shown that parenchymal sparing surgery is better than major hepatectomy in terms of higher salvageability and prolonged survival outcomes (46).

\section{Basic principle of adjuvant therapies in the context of time-to-surgical failure}

In patients with CLMs, recurrence after surgery does not mean failure of treatment. Empirically, most of the liver lesions are emerging within 2 years after the removal of primary lesion without chemotherapy during the clinical course. Although there has been scarce evidence regarding the necessity of adjuvant therapies after resection of CLM $(47,48)$, a randomized controlled trial has shown that oral adjuvant therapy reduces recurrence-free survival (RFS) rates (49), and our group also confirmed that adjuvant therapy may decrease recurrence and improve survival outcomes regardless of the chemotherapy regimens (50). Considering that prolonged chemotherapy with modern regimens such as FOLFOX or FOLFIRI may induce liver injury that can be an obstacle for repeated resection, we have adopted oral Uracil-Tegafur with Leucovorin (6 months in total) as a 1st choice for adjuvant therapy after resection of CLM.

\section{Surgical outcomes of RAS-mutated CLM}

A review of 163 patients with a known KRAS exon-2 mutation status who underwent curative resection for CLM at 2 Japanese high-volume centers revealed that $K R A S$ exon2 mutation was associated with poorer RFS, shorter time to surgical failure (TSF) (45), and poorer disease-specific survival (DSS) rates (12). KRAS exon-2 mutation showed significant association especially with lung metastases, and it was the main cause of early TSF, regardless of the sideness of primary lesions.

When reviewing 139 patients who underwent curative liver resections for CLM with a known RAS status during the last decade at Toranomon Hospital, $R A S$-mutated group showed significantly shorter TSF (34.5 vs. 14.0 months; $\mathrm{P}<0.001)$ and shorter overall survival (5-year survival rates, $60.7 \%$ vs. $39.8 \%, \mathrm{P}=0.001$ ) (Figure 3 ) in line with the previous report (12). Proportion of $R A S$-mutated cases decreased as the number of hepatectomy increases (initial hepatectomy, $42.5 \%$; 2 nd hepatectomy, $27.7 \%$; 3 rd hepatectomy, $20.0 \%$; and 4th hepatectomy, $0 \%: \mathrm{P}=0.006$ ), reflecting that unresectable recurrence is more frequent among the patients with $R A S$-mutated CLMs.

\section{Response to chemotherapy of RAS-mutated CLM}

$R A S$ status is nowadays used in our daily practice for predicting potential response to anti-EGFR antibodies. However, its utility in prediction of response to the other regimens remains unclear. In a group of patients with heavily treated with bevacizumab, careful pathological and radiological reviews have shown that $R A S$ mutation was potentially associated with poor pathologic and radiological morphologic responses (15). Although similar tendency was also observed in a Japanese population, its reproducibility has never been proven in external cohorts.

Our group previously performed comprehensive sequencing of 578 cancer-related genes and showed that MICA gene could be a potential biomarker in prediction 

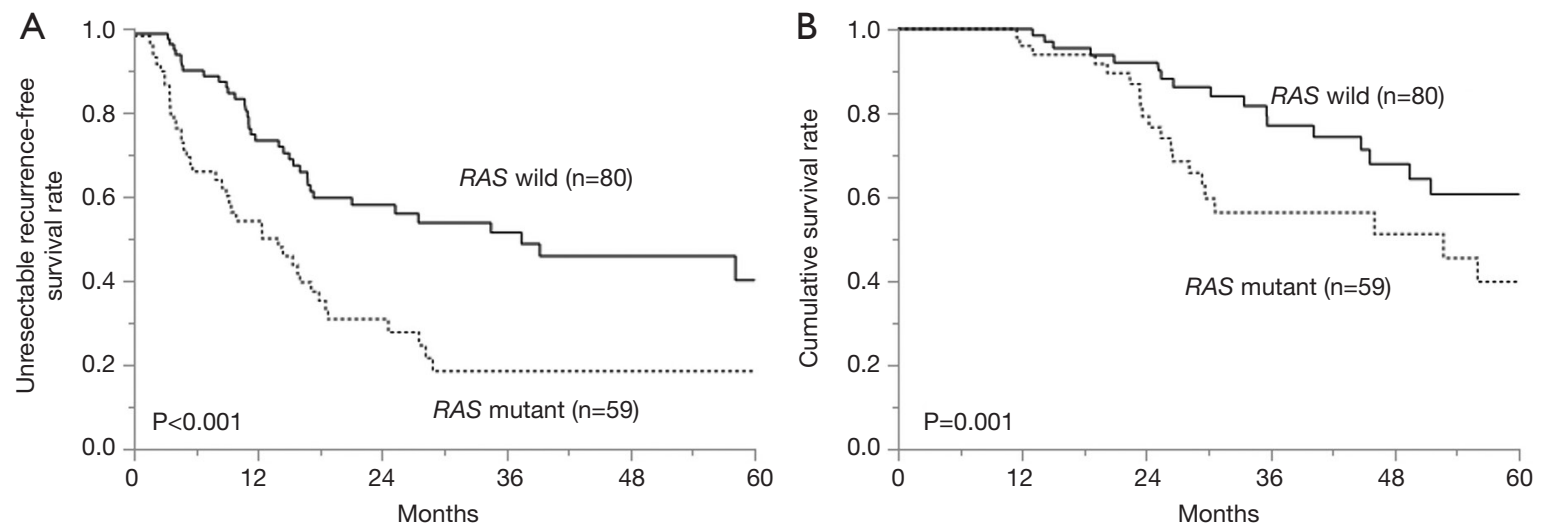

Figure 3 Survival outcomes according to $R A S$ mutational status: latest series at Toranomon Hospital. (A) Time-to-surgical failure; (B) overall survival.

of response to chemotherapy with bevacizumab and survival outcomes (51). The MICA gene is a member of the major histocompatibility complex (MHC) class I chain-related genes family, encoding a membrane-bound protein that function as a ligand to stimulate NKG2D expressed on all human natural killer and $\gamma \delta \mathrm{T}$ cells. There are 5 known variants in the MICA gene, and the study showed that MICA A5.1 variant was associated with better pathologic response (38)/morphologic response $(39,41)$ to bevacizumab, regardless of the $R A S$ mutational status. Also, MICA A5.1 was associated with a decreased risk of recurrence after resection of CLM. Since somatic mutation is quite rare in the MICA gene and very high concordance rate in the MICA variant (96.6\%) was confirmed between CLM tissue and normal liver parenchyma, these results suggested that the genetic background of the host could be a potential biomarker for CLM. Because bevacizumab has a potential to suppress angiogenesis and modulate immune environments in cancerous tissue, such genetic difference among the host influencing immune response might be associated with the efficacy of bevacizumab. Because response to chemotherapy is not determined only by the RAS mutational status of CLM, further studies would be needed including the analysis of genetic background of the host to optimize the treatment approach for the patients with $R A S$-mutated CLMs.

\section{Treatment strategy for RAS-mutated CLM: lessons from long-term survivors}

Of the 59 patients with RAS-mutated CLM treated at our institution, there were 11 patients who survived without unresectable recurrence more than 1 year from the initial hepatectomy. Although no specific clinical factors including size, number of tumors, primary sideness, primary nodal status, location of $R A S$ mutation, disease free interval from resection of primary lesion, or perioperative chemotherapy was identified as a potential predictor for survival, 4 out of the 11 patients developed recurrence during the observational period (liver, $\mathrm{n}=4$; lung, $\mathrm{n}=1$; hilar lymph node, $\mathrm{n}=1$; right adrenal gland, $\mathrm{n}=1$ ) and all of them were curatively resected.

Indeed, there has been no evidence regarding the optimal treatment approach for $R A S$-mutated CLMs. However, repeated resection seems to have survival benefit in both the $R A S$ wild cases and $R A S$ mutant cases because time-tosurgical failure (45) showed better correlation with overall survival than RFS regardless of the RAS mutations status (Figure 4). As such, aggressive surgical approach would be the only reliable strategy to maximize survival outcomes of patients with CLM irrespective of $R A S$ mutational status.

\section{Conclusions}

$R A S$ mutation is reportedly associated with oncological aggressiveness of CLMs especially with higher risk of lung metastases which precludes curative-intent treatment and determines time-to-surgical failure. Although there has been scarce evidence that additional treatment such as perioperative chemotherapy is effective for $R A S$-mutated CLMs, aggressive surgical approach seems to improve overall survival through prolonged cancer-free interval regardless of the RAS mutational status. Therefore, basic multidisciplinary approach for CLMs would be important 

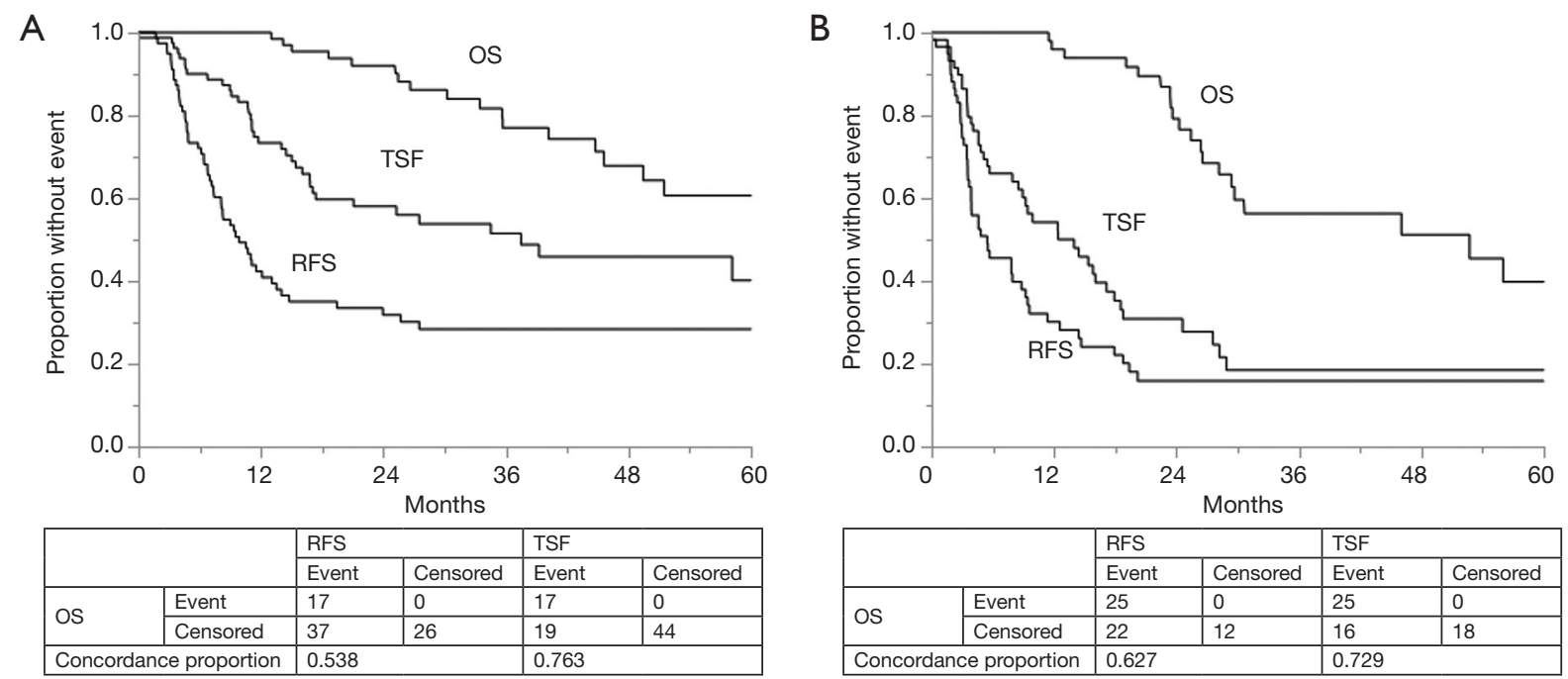

Figure 4 Comparison of survival measures stratified by RAS mutational status. (A) RAS wild (n=80); (B) RAS mutant (n=59). OS, overall survival; RFS, recurrence-free survival; TSF, time-to-surgical failure.

in both RAS wild CLMs and RAS-mutated CLMs to maximize survival outcomes.

\section{Acknowledgments}

None.

\section{Footnote}

Conflicts of Interest: The author has no conflicts of interest to declare.

Ethical Statement: The author is accountable for all aspects of the work in ensuring that questions related to the accuracy or integrity of any part of the work are appropriately investigated and resolved.

\section{References}

1. Kopetz S, Chang GJ, Overman MJ, et al. Improved survival in metastatic colorectal cancer is associated with adoption of hepatic resection and improved chemotherapy. J Clin Oncol 2009;27:3677-83.

2. Fong Y, Fortner J, Sun RL, et al. Clinical score for predicting recurrence after hepatic resection for metastatic colorectal cancer: analysis of 1001 consecutive cases. Ann Surg 1999;230:309-18; discussion 318-21.

3. Nordlinger B, Guiguet M, Vaillant JC, et al. Surgical resection of colorectal carcinoma metastases to the liver. A prognostic scoring system to improve case selection, based on 1568 patients. Association Francaise de Chirurgie. Cancer 1996;77:1254-62.

4. Rees M, Tekkis PP, Welsh FK, et al. Evaluation of longterm survival after hepatic resection for metastatic colorectal cancer: a multifactorial model of 929 patients. Ann Surg 2008;247:125-35.

5. Amikura K, Akagi K, Ogura T, et al. The RAS mutation status predicts survival in patients undergoing hepatic resection for colorectal liver metastases: The results from a genetic analysis of all-RAS. J Surg Oncol 2018;117:745-55.

6. Brudvik KW, Jones RP, Giuliante F, et al. RAS Mutation Clinical Risk Score to Predict Survival After Resection of Colorectal Liver Metastases. Ann Surg 2019;269:120-6.

7. Brudvik KW, Kopetz SE, Li L, et al. Meta-analysis of KRAS mutations and survival after resection of colorectal liver metastases. Br J Surg 2015;102:1175-83.

8. Denbo JW, Yamashita S, Passot G, et al. RAS Mutation Is Associated with Decreased Survival in Patients Undergoing Repeat Hepatectomy for Colorectal Liver Metastases. J Gastrointest Surg 2017;21:68-77.

9. Karagkounis G, Torbenson MS, Daniel HD, et al. Incidence and prognostic impact of KRAS and BRAF mutation in patients undergoing liver surgery for colorectal metastases. Cancer 2013;119:4137-44.

10. Okuno M, Goumard C, Kopetz S, et al. RAS Mutation 
is Associated with Unsalvageable Recurrence Following Hepatectomy for Colorectal Cancer Liver Metastases. Ann Surg Oncol 2018;25:2457-66.

11. Passot G, Kim BJ, Glehen O, et al. Impact of RAS Mutations in Metastatic Colorectal Cancer After Potentially Curative Resection: Does Site of Metastases Matter? Ann Surg Oncol 2018;25:179-87.

12. Shindoh J, Nishioka Y, Yoshioka R, et al. KRAS Mutation Status Predicts Site-Specific Recurrence and Survival After Resection of Colorectal Liver Metastases Irrespective of Location of the Primary Lesion. Ann Surg Oncol 2016;23:1890-6.

13. Stremitzer S, Stift J, Gruenberger B, et al. KRAS status and outcome of liver resection after neoadjuvant chemotherapy including bevacizumab. Br J Surg 2012;99:1575-82.

14. Vauthey JN, Zimmitti G, Kopetz SE, et al. RAS mutation status predicts survival and patterns of recurrence in patients undergoing hepatectomy for colorectal liver metastases. Ann Surg 2013;258:619-26; discussion 626-7.

15. Zimmitti G, Shindoh J, Mise Y, et al. RAS mutations predict radiologic and pathologic response in patients treated with chemotherapy before resection of colorectal liver metastases. Ann Surg Oncol 2015;22:834-42.

16. Margonis GA, Buettner S, Andreatos N, et al. Association of BRAF Mutations With Survival and Recurrence in Surgically Treated Patients With Metastatic Colorectal Liver Cancer. JAMA Surg 2018;153:e180996.

17. Kawaguchi Y, Lillemoe HA, Panettieri E, et al. Conditional Recurrence-Free Survival after Resection of Colorectal Liver Metastases: Persistent Deleterious Association with RAS and TP53 Co-Mutation. J Am Coll Surg 2019. [Epub ahead of print].

18. Mizuno T, Cloyd JM, Vicente D, et al. SMAD4 gene mutation predicts poor prognosis in patients undergoing resection for colorectal liver metastases. Eur J Surg Oncol 2018;44:684-92.

19. Nakayama I, Shinozaki E, Matsushima T, et al. Retrospective study of RAS/PIK3CA/BRAF tumor mutations as predictors of response to first-line chemotherapy with bevacizumab in metastatic colorectal cancer patients. BMC Cancer 2017;17:38.

20. Nash GM, Gimbel M, Cohen AM, et al. KRAS mutation and microsatellite instability: two genetic markers of early tumor development that influence the prognosis of colorectal cancer. Ann Surg Oncol 2010;17:416-24.

21. Nash GM, Gimbel M, Shia J, et al. KRAS mutation correlates with accelerated metastatic progression in patients with colorectal liver metastases. Ann Surg Oncol
2010;17:572-8.

22. Tie J, Lipton L, Desai J, et al. KRAS mutation is associated with lung metastasis in patients with curatively resected colorectal cancer. Clin Cancer Res 2011;17:1122-30.

23. Margonis GA, Sasaki K, Andreatos N, et al. KRAS Mutation Status Dictates Optimal Surgical Margin Width in Patients Undergoing Resection of Colorectal Liver Metastases. Ann Surg Oncol 2017;24:264-71.

24. Brudvik KW, Mise Y, Chung MH, et al. RAS Mutation Predicts Positive Resection Margins and Narrower Resection Margins in Patients Undergoing Resection of Colorectal Liver Metastases. Ann Surg Oncol 2016;23:2635-43.

25. Adam R, De Gramont A, Figueras J, et al. The oncosurgery approach to managing liver metastases from colorectal cancer: a multidisciplinary international consensus. Oncologist 2012;17:1225-39.

26. Van Cutsem E, Cervantes A, Adam R, et al. ESMO consensus guidelines for the management of patients with metastatic colorectal cancer. Ann Oncol 2016;27:1386-422.

27. Adams RB, Aloia TA, Loyer E, et al. Selection for hepatic resection of colorectal liver metastases: expert consensus statement. HPB (Oxford) 2013;15:91-103.

28. Beppu T, Sakamoto Y, Hasegawa K, et al. A nomogram predicting disease-free survival in patients with colorectal liver metastases treated with hepatic resection: multicenter data collection as a Project Study for Hepatic Surgery of the Japanese Society of Hepato-Biliary-Pancreatic Surgery. J Hepatobiliary Pancreat Sci 2012;19:72-84.

29. Makuuchi M, Kosuge T, Takayama T, et al. Surgery for small liver cancers. Semin Surg Oncol 1993;9:298-304.

30. Kobayashi Y, Kiya Y, Sugawara T, et al. Expanded Makuuchi's criteria using estimated indocyanine green clearance rate of future liver remnant as a safety limit for maximum extent of liver resection. HPB (Oxford) 2019. [Epub ahead of print].

31. Omichi K, Yamashita S, Cloyd JM, et al. Portal Vein Embolization Reduces Postoperative Hepatic Insufficiency Associated with Postchemotherapy Hepatic Atrophy. J Gastrointest Surg 2018;22:60-7.

32. Tani K, Shindoh J, Takamoto T, et al. Kinetic Changes in Liver Parenchyma After Preoperative Chemotherapy for Patients with Colorectal Liver Metastases. J Gastrointest Surg 2017;21:813-21.

33. Shindoh J, Kobayashi Y, Kinowaki K, et al. Dynamic Changes in Normal Liver Parenchymal Volume During Chemotherapy for Colorectal Cancer: Liver Atrophy as 
an Alternate Marker of Chemotherapy-Associated Liver Injury. Ann Surg Oncol 2019. [Epub ahead of print].

34. Kishi Y, Zorzi D, Contreras CM, et al. Extended preoperative chemotherapy does not improve pathologic response and increases postoperative liver insufficiency after hepatic resection for colorectal liver metastases. Ann Surg Oncol 2010;17:2870-6.

35. Shindoh J, Tzeng CW, Aloia TA, et al. Optimal future liver remnant in patients treated with extensive preoperative chemotherapy for colorectal liver metastases. Ann Surg Oncol 2013;20:2493-500.

36. Aloia TA, Zorzi D, Abdalla EK, et al. Two-surgeon technique for hepatic parenchymal transection of the noncirrhotic liver using saline-linked cautery and ultrasonic dissection. Ann Surg 2005;242:172-7.

37. Karoui M, Penna C, Amin-Hashem M, et al. Influence of preoperative chemotherapy on the risk of major hepatectomy for colorectal liver metastases. Ann Surg 2006;243:1-7.

38. Blazer DG 3rd, Kishi Y, Maru DM, et al. Pathologic response to preoperative chemotherapy: a new outcome end point after resection of hepatic colorectal metastases. J Clin Oncol 2008;26:5344-51.

39. Chun YS, Vauthey JN, Boonsirikamchai P, et al. Association of computed tomography morphologic criteria with pathologic response and survival in patients treated with bevacizumab for colorectal liver metastases. JAMA 2009;302:2338-44.

40. Nishioka Y, Shindoh J, Yoshioka R, et al. Radiological Morphology of Colorectal Liver Metastases after Preoperative Chemotherapy Predicts Tumor Viability and Postoperative Outcomes. J Gastrointest Surg 2015;19:1653-61.

41. Shindoh J, Loyer EM, Kopetz S, et al. Optimal morphologic response to preoperative chemotherapy: an alternate outcome end point before resection of hepatic colorectal metastases. J Clin Oncol 2012;30:4566-72.

42. Nishioka Y, Shindoh J, Yoshioka R, et al. Clinical Impact of Preoperative Chemotherapy on Microscopic Cancer Spread Surrounding Colorectal Liver Metastases. Ann

Cite this article as: Shindoh J. Japanese experience with hepatic resection of $K R A S$-mutated colorectal liver metastases. Chin Clin Oncol 2019;8(5):47. doi:10.21037/cco.2019.08.07
Surg Oncol 2017;24:2326-33.

43. Andreou A, Aloia TA, Brouquet A, et al. Margin status remains an important determinant of survival after surgical resection of colorectal liver metastases in the era of modern chemotherapy. Ann Surg 2013;257:1079-88.

44. Shindoh J, Makuuchi M, Matsuyama Y, et al. Complete removal of the tumor-bearing portal territory decreases local tumor recurrence and improves disease-specific survival of patients with hepatocellular carcinoma. J Hepatol 2016;64:594-600.

45. Oba M, Hasegawa K, Matsuyama Y, et al. Discrepancy between recurrence-free survival and overall survival in patients with resectable colorectal liver metastases: a potential surrogate endpoint for time to surgical failure. Ann Surg Oncol 2014;21:1817-24.

46. Mise Y, Aloia TA, Brudvik KW, et al. Parenchymal-sparing Hepatectomy in Colorectal Liver Metastasis Improves Salvageability and Survival. Ann Surg 2016;263:146-52.

47. Nordlinger B, Rougier P, Arnaud JP, et al. Adjuvant regional chemotherapy and systemic chemotherapy versus systemic chemotherapy alone in patients with stage IIIII colorectal cancer: a multicentre randomised controlled phase III trial. Lancet Oncol 2005;6:459-68.

48. Nordlinger B, Sorbye H, Glimelius B, et al. Perioperative FOLFOX4 chemotherapy and surgery versus surgery alone for resectable liver metastases from colorectal cancer (EORTC 40983): long-term results of a randomised, controlled, phase 3 trial. Lancet Oncol 2013;14:1208-15.

49. Hasegawa K, Saiura A, Takayama T, et al. Adjuvant Oral Uracil-Tegafur with Leucovorin for Colorectal Cancer Liver Metastases: A Randomized Controlled Trial. PLoS One 2016;11:e0162400.

50. Nishioka Y, Moriyama J, Matoba S, et al. Prognostic Impact of Adjuvant Chemotherapy after Hepatic Resection for Synchronous and Early Metachronous Colorectal Liver Metastases. Dig Surg 2018;35:187-95.

51. Nishioka Y, Shindoh J, Inagaki Y, et al. Host MICA Polymorphism as a Potential Predictive Marker in Response to Chemotherapy for Colorectal Liver Metastases. Dig Dis 2018;36:437-45. 